\title{
InterPreT Cancer Survival: A dynamic web interactive prediction cancer survival tool for health-care professionals and cancer epidemiologists
}

\author{
Authors: Sarwar Islam Mozumder (sarwar.islam@le.ac.uk), Paul W Dickman ${ }^{b}$ \\ (paul.dickman@ki.se), MarkJ Rutherforda (mjr40@le.ac.uk),Paul C Lamberta,b (pl4@le.ac.uk) \\ a Biostatistics Research Group, Department of Health Sciences, University of Leicester, UK \\ ${ }^{b}$ Department of Medical Epidemiology \& Biostatistics, Karolinska Institutet, Stockholm, Sweden
}

Correspondence: Sarwar Islam Mozumder, Biostatistics Research Group, Department of Health Sciences, College of Life Sciences, University of Leicester, Centre for Medicine, University Road, Leicester, LE1 7RH, UK.

E-mail: sarwar.islam@le.ac.uk. Tel: +44116229 7255.

Financial support: This research did not receive any specific grant from funding agencies in the public, commercial, or not-for-profit sectors.

Conflict of interest disclosure statement: No conflicts of interest to be declared.

Number of tables/figures: 0 tables and 4 figures.

Word count: 248 (abstract) +2982 (main) +51 (highlights)

Availability: The online interactive cancer survival prediction tool, InterPreT, can be accessed via http://interpret.le.ac.uk. The web tool is compatible with most web browsers excluding Internet Explorer (e.g. Chrome, Firefox, Edge and Safari). We highly encourage readers to use the tool whilst reading the paper. 


\section{Abstract}

Background: There are a variety of ways for quantifying cancer survival with each measure having advantages and disadvantages. Distinguishing these measures and how they should be interpreted has led to confusion among scientists, the media, health care professionals and patients. This motivates the development of tools to facilitate communication and interpretation of these statistics.

Methods: "InterPreT Cancer Survival" is a newly developed, publicly available, online interactive cancer survival tool targeted towards health-care professionals and epidemiologists (http://interpret.le.ac.uk). It focuses on the correct interpretation of commonly reported cancer survival measures facilitated through the use of dynamic interactive graphics. Statistics presented are based on parameter estimates obtained from flexible parametric relative survival models using large population-based English registry data containing information on survival across 6 cancer sites; Breast, Colon, Rectum, Stomach, Melanoma and Lung.

Results: Through interactivity, the tool improves understanding of various measures and how survival or mortality may vary by age and sex. Routine measures of cancer survival are reported, however, individualised estimates using crude probabilities are advocated, which is more appropriate for patients or health care professionals. The results are presented in various interactive formats facilitating understanding of individual risk and differences between various measures.

Conclusions: "InterPreT Cancer Survival" is presented as an educational tool which engages the user through interactive features to improve the understanding of commonly reported cancer survival statistics. The tool has received positive feedback from a Cancer Research UK patient sounding board and there are further plans to incorporate more disease characteristics, e.g. stage.

Keywords: cancer survival, flexible parametric survival model, crude probability of death, net survival, interactive web-tool. 


\section{Introduction}

Researchers are often interested in quantifying patient survival after a cancer diagnosis in the entire population. This is explored in large population-based studies to monitor and evaluate effectiveness of patient care to give estimates representative of the whole population [1]. Often, survival in the hypothetical scenario where the cancer of interest is the only possible cause of death, i.e. net survival, is estimated. This allows comparison of survival over time between different population groups which may vary in mortality from other causes.

Net survival is usually age standardised to give averages over the whole study population. Although age standardisation is useful for reporting a single aggregated summary statistic and making comparisons, it hides variation in net survival across age that exists for most cancers $[2,3]$.

Another consequence of reporting (standardised) net survival, is the tendency to misinterpret it as observed survival. Net survival is a cancer-specific estimate which removes other cause mortality and therefore does not represent individual patient survival in the real-world. However, despite these warnings, when communicated to the public, this is often not portrayed [4]. To present information that is more relevant for the patient, the real-world, or crude, probability of death in the presence of dying from other competing causes is more appropriate [5]

To support the use of interactive tools that aid risk communication, Trevena et al. [6] conducted a systematic search to explore impact and effectiveness of alternative communication tools on understanding risk. They concluded that, presenting information in 
alternative formats, which included computer-based approaches and interactivity (i.e. features that allow the user to control and navigate through graphs by making input alterations), substantially increased comprehension of individual risk. Many also advocate presenting health statistics as natural frequencies, as opposed to probabilities or conditional probabilities, as they are better understood by physicians and non-statisticians [7, 8, 9]. Although there are several online tools which present health statistics this way, there remain a shortage of tools that allow interactive comparisons between different measures [10]. We introduce InterPreT as an educational tool for physicians and epidemiologists and to facilitate communication of cancer statistics to patients and the general public.

\section{Material \& Methods}

\subsection{Data}

InterPreT uses English cancer registry data obtained from the National Cancer Registration and Analysis Service (NCRAS) run by Public Health England. The data contains information on age, sex and survival in days for patients diagnosed with 6 different cancers from 1995 to 2013. Analysis was restricted to patients aged 40 to 90 years old at diagnosis. English population mortality life tables, stratified by age, sex and calendar year were obtained from the Cancer Survival Group at the London School of Hygiene and Tropical Medicine (http://csg.Ishtm.ac.uk/tools-analysis/uk-life-tables). 


\subsection{Cancer Survival Measures}

Pohar Perme et al. [11] summarise the different metrics available on cancer survival and concludes that, when interest is in patient health-related decisions, crude mortality probabilities are more appropriate $[12,13]$. However, researchers are sometimes interested in making unbiased comparisons in cancer-specific survival between different populations which can potentially differ in terms of the mortality associated with other causes. In this case, net survival is of interest. An overview of the measures reported in InterPret are summarised below.

\subsubsection{Net survival}

Net survival is interpreted as the survival probability in the hypothetical world where one can only die from the cancer of interest. It can be estimated in either a cause-specific, or relative survival framework. The relative survival approach is more popular in large population based cancer studies and has been applied in many scenarios using a flexible parametric relative survival modelling approach (see section 2.3.2).

\subsubsection{Expected survival}

Expected survival, $S_{i}^{*}(t)$, is calculated directly from population mortality life tables matched by age, sex and calendar year from expected mortality rates, $h_{i}^{*}(t)$. Hence, expected survival gives the chance of being alive for a person of the same calendar year, age and sex in the general population (who are assumed to be free of the cancer under study). 


\subsubsection{Observed survival}

Observed survival, $S_{i}(t)$, is the probability that a patient is still alive at time $t$ following a cancer diagnosis, where they are at risk of dying from cancer or any other causes. This is the most basic summary on patient survival, however, it does not distinguish mortality due to cancer from mortality due to other causes.

\subsubsection{Relative survival}

Relative survival, $R_{i}(t)$, can be interpreted as net survival under certain assumptions [14]. The first involves conditional independence between deaths associated with the cancer of interest and non-cancer related mortality. The second requires that expected mortality rates, reflecting the non-cancer mortality, are appropriate for the cancer study population and are stratified by relevant covariates. It is calculated as a ratio between observed survival and expected survival, i.e., $R_{i}(t)=S_{i}(t) / S_{i}^{*}(t)$.

\subsubsection{Crude probability of death}

The crude probability of death partitions all-cause probability of death into death due to cancer and other causes. This measure has a real-world interpretation and is useful for treatmentrelated decisions at the individual-level or for planning future health-care services.

\subsubsection{Conditional survival}

Conditional survival probabilities are also presented for all the above measures. This gives the probability of survival, or death, at $t$ years given that the patient has already survived $t_{0}$ years after diagnosis. 


\subsection{Statistical methods}

\subsubsection{Period analysis}

Relative survival is often reported over a time-period, for example, they can be given at 1-, 5and 10 -years after diagnosis. In a typical relative survival analysis, all available information on the survival experience of patients diagnosed with cancer are usually included. For instance, if relative survival is reported at 5 years since diagnosis, information on a mixture of patients diagnosed recently and over 5 years ago would be incorporated. However, the survival experience of patients diagnosed recently is likely to be different to those diagnosed more than 5 years ago due to factors such as advancements in cancer treatment. As a result, cancer patients diagnosed recently are likely to have an improved survival experience over-time due to better health-care. Therefore, reporting estimates from analyses based on patients that were diagnosed at least 5 or 10 years ago are subject to the underestimation of cancer survival probabilities. Furthermore, it is likely that cancer registry data will be published a year or two later after the study. This leads to a further time-lag between cancer diagnosis and evaluation

In order to obtain more up-to-date estimates on long-term cancer patient survival, the period survival analysis approach is adopted, as first introduced by Brenner and Gefeller [15]. This approach restricts analysis to the survival experience in the most recent years (defined as a period window) and so, those diagnosed more early on in calendar time with a short-term survival are excluded from the analysis [16].

\subsubsection{Flexible parametric relative survival models}

A popular approach beyond the Cox model for survival analysis was introduced by Royston and Parmar [17] which allow for more flexibility and better capture the behaviour of real-world 
datasets. Using expected mortality rates, these models were later extended for relative survival which are used extensively in large population-based studies to obtain predictions that quantify cancer patient survival [18].

We calculate crude probabilities of death after fitting a flexible parametric relative survival model and therefore partition crude probability of death due to any cause (i.e. 1 minus observed survival), $F_{\text {all,i }}(t)$, into the crude probability of death due to cancer, $F_{\text {cancer }, i}(t)$, and crude probability of death due to other causes, $F_{\text {other }, i}(t)[19]$.

Flexible parametric relative survival models are fitted to the data using the stpm2 command in Stata [20]. Individual models are fitted for each sex with continuous age at diagnosis as the only included covariate. This is assumed to have a non-linear effect using restricted cubic splines. Models were also fitted under a period analysis, where up-to-date estimates were obtained using a period window of 01 January 2013 to 31 December 2015 [21]. Only the model parameters and details about the number and location of knots for the spline variables are exported and stored on the online servers, thereby preserving the privacy of sensitive information. Further details of the models can be found at http://interpret.le.ac.uk/methods.php.

\subsection{Development}

\subsubsection{Data-driven documents (D3)}

Many tools exist that allow users to create interactive visualisations of data within the webenvironment which combine a variety of technologies. At the core are Hypertext Markup Language (HTML) for structuring the web-page, Cascading Style Sheets (CSS) for web-page 
aesthetics and JavaScript for creating interactive content $[22,23]$. The co-operation of such technologies are made possible through the document object model (DOM) which is a native representation behind every web-page that allows for reference and manipulation of online content. Bostock et al. [24] introduces Data-Driven Documents, or, d3.js, as a "representation transparent approach to visualisation for the web". D3.js is a tool which is available as a JavaScript library that combines the above triad of technologies, including additional ones, such as scalar vector graphics (SVG), for creating dynamic interactive visualisations.

Using the increasingly popular d3. j s library, the educational online interactive tool for cancer survival, InterPreT Cancer Survival was developed. The tool's primary focus is on the correct interpretation of commonly reported cancer survival measures facilitated through the use of dynamic interactive graphics allowing users to make contrasts between the various measures.

\subsubsection{Interactive Features}

The web-tool presents cancer survival in a language that is accessible for users from various backgrounds. Statistics are interpreted as natural frequencies, i.e. "out of 100", to allow users to distinguish between the various metrics. Summary probability tables are presented providing a snapshot of survival at 1, 5 and 10 years after diagnosis. A visual representation of these natural frequencies are available using people charts for all measures. By default these are illustrated for patients 5 years after diagnosis, but can be changed by users for 1 to 10 years from diagnosis. Alternatively, line charts are available on both survival and mortality as well as stack charts for crude probabilities of death. 
All plots are dynamic and probabilities are visible on mouse-over. Users may select or de-select cancer measures of interest for specific comparisons. A fix check-box is also available to save statistics for a particular set of characteristics to enable visual comparisons with other patient groups. A slider allows the user to change the age of the patient which instantaneously updates the plots, facilitating observations on the changes in cancer survival for older or younger patients.

Conditional probabilities may also be displayed by dragging the $y$-axis across time (figure 1 ). The presentation of conditional probabilities is a particularly useful and powerful interactive component of the InterPreT Cancer Survival web-tool from a prognosis point of view. As highlighted by Bostrom et al. [25], the portrayal of changes in risk under different "what if" scenarios is one of the many advantages of introducing interactive features in visualisations [26]. For example, in this particular case, by dragging the $y$-axis, the user can explore the scenario of "what if I was still alive after 3 years, how would my survival probability change?"

\section{Results: An illustration of using I nterPreT to distinguish between net and crude measures}

InterPreT is catered towards understanding differences in interpretation between various cancer survival measures. For example, net survival is often incorrectly reported as observed survival, or misinterpreted as the crude probability of death due to cancer. Understanding 
these differences is demonstrated using InterPreT. We encourage following the example below using the tool online.

We begin by choosing a 45 year old female with breast cancer and focus on comparing her net survival probability with an 85 year old patient. Using the plot and text descriptions in InterPreT (see figure 2), we can see that, 80 out of 10045 year old women with breast cancer are likely to still be alive 10 years after diagnosis. Whereas, for 85 year olds, 45 out of 100 women are likely to still be alive. However, these probabilities only take into account the chance of dying from cancer and excludes the possibility of dying from anything else. It therefore refers to some hypothetical scenario where cancer is the only cause of death. To see how and why this differs from their actual risk of dying from cancer, i.e., their crude probability of death due to cancer, we switch to stack charts by choosing probabilities in terms of mortality from the drop down menu (see figure 3). For 45 year old female cancer patients, we can visualise their crude probability of death due to any cause and partition this into the probability of dying of cancer and other causes. Younger patients are naturally less likely to die from other causes ( 2 out of 100 by 10 years), therefore it is not surprising that their net probability of death, i.e., 1 minus net survival, is similar to their crude probability of death due to cancer. The distinction is more apparent as we drag the slider across for older patients. As the patient's age is increased, we see a larger proportion dying from other causes, as represented by the increasing area of the partition for other causes. In contrast, the crude probability of death due to cancer increases more slowly compared to the net probability of death since, in reality, a lot of these older patients are more likely to die of other causes. As we get to 85 year old patients, a clear difference is observed between the net probability of death and crude probability of death due to cancer. The real-world probability of dying from cancer is lower (38 out of 100) 
and a higher proportion of the patient's mortality is attributable to other causes (51 out of 100). This can also be observed by switching to the people charts where a similar comparison can be made (see figure 4).

\section{Discussion}

This paper introduces an interactive online tool, InterPreT, which aims to communicate complicated, commonly reported cancer survival statistics to professionals such as clinicians and epidemiologists. The primary purpose is to aid communication and interpretation of these measures and facilitate easy comparisons across different patient characteristics through interactive features. Although aimed primarily towards clinicians and epidemiologists, as the tool is publicly available, patients will also have access to InterPreT to aid their understanding of various metrics that describe the impact of their diagnosed cancer. Therefore, a disclaimer has been placed to highlight the intended use of InterPreT, offering advice on support and sources of further information.

Due to the lack of current publicly available interactive web-tools for communicating risk, there is very little literature on the efficacy of web-based interactive graphics on the improvement in the understanding of risk [27]. Therefore, due to the lack of qualitative studies that address this, it is uncertain whether making such tools available for the public improves the understanding of various cancer survival measures. However, we have informally evaluated InterPreT Cancer Survival's impact on improving the understanding of a variety of cancer survival statistics by consulting a panel of patients via the Cancer Research UK patient sounding board (see section 4.1). It has also been user-tested by a number of epidemiologists and clinicians. Generally, feedback from these users indicated that InterPreT Cancer 
Survival succeeded as an educational tool that could primarily be used to aid interpretation, or as supplemental material to help others distinguish between various cancer survival measures. Furthermore, the components and language incorporated into the web-tool have all been shown in previous studies to contribute to the improvement in the understanding and interpretation of information presented as probabilities $[7,8,9]$.

The problem of what to present and communicate to patients is paradoxical in nature due to the awkwardness in interpretation of prognostic-relevant measures on the survival scale. Hagerty et al. [28] reviewed literature specifically in relation to the communication of patient prognosis in the context of cancer care. It was found that, patients in the early stages of their cancer welcomed detailed information on their prognosis which are publicly available. However, impact of prognosis communication is unclear for patients with advanced cancers, since prognosis in such cases are not so openly discussed. Therefore, the appropriate communication of prognosis in such cases was not obvious and requires further evaluation.

\subsection{Cancer Research UK patient sounding board}

To evaluate the tool's suitability for patient-use, a Cancer Research UK patient sounding board was consulted. Overall, patients were keen on also having access to tools available to healthcare professionals. This meant that they could themselves grasp some understanding on the cancer statistics that they were presented with as opposed to relying on the vague explanations usually provided. Ease in the use of InterPreT was a feature that stood out to the patients and the interactivity of being able to see the change in survival across age and easily make comparisons was well received. Although some patients agreed that the tool was an informative way to communicate death and present information that they wanted available, 
others pointed out that this perspective may change because of the language of interpretation behind these measures. For example, "crude probabilities of death" is the metric that is most appropriate for a patient when determining their prognostic outcome and making treatment decisions. However, this terminology was considered to be unsuitable for patient communication due to the use of the word "death", whereas more positive language, such as "survival" or "alive", are preferred. Presenting cancer statistics as death probabilities is undesirable, which, in this case is unavoidable due to an awkward interpretation on the survival scale [29]. In this respect, the language used in other aspects of the tool has been tailored in consideration of how a patient may react to the information and directly affected users are given resources for support. This also potentially motivates for an alternative version of the web-tool, solely targeted towards patient-use.

\subsection{Conclusion}

We have developed an interactive online educational tool to facilitate interpretation of a variety of cancer survival statistics. However, InterPreT currently only includes basic patient characteristics in the model such as age and sex. In order to operate as a complete prognostic prediction tool with patient relevant predictions, other important disease characteristics, such as stage at diagnosis and grade of tumour, need to be included in the model. Therefore, a future version targeted towards patients incorporating further information may be developed and validated for accurate and more individualised predictions on patient prognosis whilst remaining fully interactive. 


\section{Acknowledgments}

The authors are grateful to CRUK for granting access to the patient sounding board and contributing feedback on InterPreT's user-interface. 


\section{References}

[1] P. W. Dickman and H.-O. Adami, "Interpreting trends in cancer patient survival." J Intern Med, vol. 260, no. 2, pp. 103-117, Aug 2006. [Online]. Available: http://dx.doi.org/10.1111/j.1365-2796.2006.01677.x

[2] E. J. A. Morris, F. Sandin, P. C. Lambert, F. Bray, Å. Klint, K. Linklater, D. Robinson, L. PåhIman, L. Holmberg, and H. Møller, "A population-based comparison of the survival of patients with colorectal cancer in England, Norway and Sweden between 1996 and 2004." Gut, vol. 60, no. 8, pp. 1087-1093, Aug 2011. [Online]. Available: http://dx.doi.org/10.1136/gut.2010.229575

[3] L. Holmberg, D. Robinson, F. Sandin, F. Bray, K. M. Linklater, A. Klint, P. C. Lambert, J. Adolfsson, F. C. Hamdy, J. Catto, and H. Møller, "A comparison of prostate cancer survival in England, Norway and Sweden: a population-based study." Cancer Epidemiol, vol. 36, no. 1, pp. e7-12, Feb 2012. [Online]. Available: http://dx.doi.org/10.1016/j.canep.2011.08.001

[4] M. Quaresma, M. P. Coleman, and B. Rachet, "40-year trends in an index of survival for all cancers combined and survival adjusted for age and sex for each cancer in England and Wales, 1971-2011: a population-based study." Lancet, vol. 385, pp. 1206-1218, Dec 2014. [Online]. Available: http://dx.doi.org/10.1016/S0140-6736(14)61396-9

[5] E. J. Feuer, M. Lee, A. B. Mariotto, K. A. Cronin, S. Scoppa, D. F. Penson, M. Hachey, L. Cynkin, G. A. Carter, D. Campbell, A. Percy-Laurry, Z. Zou, D. Schrag, and B. F. Hankey, "The Cancer Survival Query System: making survival estimates from the Surveillance, Epidemiology, and End Results program more timely and relevant for recently diagnosed patients." Cancer, vol. 118, no. 22, pp. 5652-5662, Nov 2012. [Online]. Available: http://dx.doi.org/10.1002/cncr.27615

[6] L. J. Trevena, A. Barratt, P. Butow, and P. Caldwell, "A systematic review on communicating with patients about evidence," Journal of evaluation in clinical practice, vol. 12, no. 1, pp. 13-23, 2006.

[7] G. Gigerenzer, W. Gaissmaier, E. Kurz-Milcke, L. M. Schwartz, and S. Woloshin, "Helping doctors and patients make sense of health statistics," Psychological science in the public interest, vol. 8, no. 2, pp. 53-96, 2007.

[8] G. Gigerenzer and A. Edwards, "Simple tools for understanding risks: from innumeracy to insight." BMJ, vol. 327, no. 7417, pp. 741-744, Sep 2003. [Online]. Available: http://dx.doi.org/10.1136/bmj.327.7417.741

[9] G. Naik, H. Ahmed, and A. G. Edwards, "Communicating risk to patients and the public," BrJ Gen Pract, vol. 62, no. 597, pp. 213-216, 2012.

[10] B. A. Rabin, B. Gaglio, T. Sanders, L. Nekhlyudov, J. W. Dearing, S. Bull, R. E. Glasgow, and A. Marcus, "Predicting cancer prognosis using interactive online tools: a systematic review and implications for cancer care providers." Cancer Epidemiol Biomarkers Prev, vol. 22, no. 10, pp. 1645-1656, Oct 2013. [Online]. Available: http://dx.doi.org/10.1158/1055-9965.EPI-13-0513

[11] M. Pohar Perme, J. Estève, and B. Rachet, "Analysing population-based cancer survival - settling the controversies." BMC cancer, vol. 16, p. 933, Dec 2016.

[12] P. C. Lambert, P. W. Dickman, C. P. Nelson, and P. Royston, "Estimating the crude probability of death due to cancer and other causes using relative survival models," Stat Med, vol. 29, pp. $885-895,2010$.

[13] S. Eloranta, J. Adolfsson, P. C. Lambert, O. Stattin, Pär and Akre, T. M.-L. Andersson, and P. W. Dickman, "How can we make cancer survival statistics more useful for patients and 
clinicians: An illustration using localized prostate cancer in sweden." Cancer Causes Control, vol. 24, pp. 505-515, Jan 2013. [Online]. Available: http://dx.doi.org/10.1007/s10552-0120141-5

[14] P. C. Lambert, P. W. Dickman, and M. J. Rutherford, "Comparison of approaches to estimating age-standardized net survival," BMC Med Res Methodol, vol. 15, p. 64, 2015.

[15] H. Brenner and O. Gefeller, "An alternative approach to monitoring cancer patient survival," Cancer, vol. 78, pp. 2004-2010, 1996.

[16] L. Jansen, T. Hakulinen, and H. Brenner, "Study populations for period analyses of cancer survival." Br J Cancer, vol. 108, no. 3, pp. 699-707, Feb 2013. [Online]. Available: http://dx.doi.org/10.1038/bjc.2013.14

[17] P. Royston and M. K. B. Parmar, "Flexible parametric proportional-hazards and proportional-odds models for censored survival data, with application to prognostic modelling and estimation of treatment effects." Statistics in Medicine, vol. 21, no. 15, pp. 2175-2197, Aug 2002. [Online]. Available: http://dx.doi.org/10.1002/sim.1203

[18] C. P. Nelson, P. C. Lambert, I. B. Squire, and D. R. Jones, "Flexible parametric models for relative survival, with application in coronary heart disease." Stat Med, vol. 26, no. 30, pp. 5486-5498, Dec 2007. [Online]. Available: http://dx.doi.org/10.1002/sim.3064

[19] P. C. Lambert, P. W. Dickman, C. L. Weston, and J. R. Thompson, "Estimating the cure fraction in population-based cancer studies by using finite mixture models," Journal of the Royal Statistical Society, Series C, vol. 59, pp. 35-55, 2010.

[20] P. C. Lambert and P. Royston, "Further development of flexible parametric models for survival analysis," The Stata Journal, vol. 9, pp. 265-290, 2009.

[21] H. Brenner and T. Hakulinen, "Up-to-date cancer survival: period analysis and beyond." Int J Cancer, vol. 124, no. 6, pp. 1384-1390, Mar. 2009.

[22] D. Flanagan, JavaScript: the definitive guide. " O’Reilly Media, Inc.", 2006.

[23] H. W. Lie and B. Bos, Cascading style sheets: Designing for the web. Addison-Wesley Professional, 2005.

[24] M. Bostock, V. Ogievetsky, and J. Heer, "D³ data-driven documents," IEEE transactions on visualization and computer graphics, vol. 17, no. 12, pp. 2301-2309, 2011.

[25] A. Bostrom, L. Anselin, and J. Farris, "Visualizing seismic risk and uncertainty," Annals of the New York Academy of Sciences, vol. 1128, no. 1, pp. 29-40, 2008.

[26] V. J. Strecher, T. Greenwood, C. Wang, and D. Dumont, "Interactive multimedia and risk communication," JNCl Monographs, vol. 1999, no. 25, pp. 134-139, 1999.

[27] L. J. Trevena, B. J. Zikmund-Fisher, A. Edwards, W. Gaissmaier, M. Galesic, P. K. Han, J. King, M. L. Lawson, S. K. Linder, I. Lipkus et al., "Presenting quantitative information about decision outcomes: a risk communication primer for patient decision aid developers," BMC medical informatics and decision making, vol. 13, no. 2, p. S7, 2013.

[28] R. Hagerty, P. N. Butow, P. Ellis, S. Dimitry, and M. Tattersall, "Communicating prognosis in cancer care: a systematic review of the literature," Annals of Oncology, vol. 16, no. 7, pp. 1005-1053, 2005.

[29] K. A. Cronin and E. J. Feuer, "Cumulative cause-specific mortality for cancer patients in the presence of other causes: a crude analogue of relative survival." Statistics in Medicine, vol. 19, no. 13, pp. 1729-1740, 2000. 


\section{Figures}

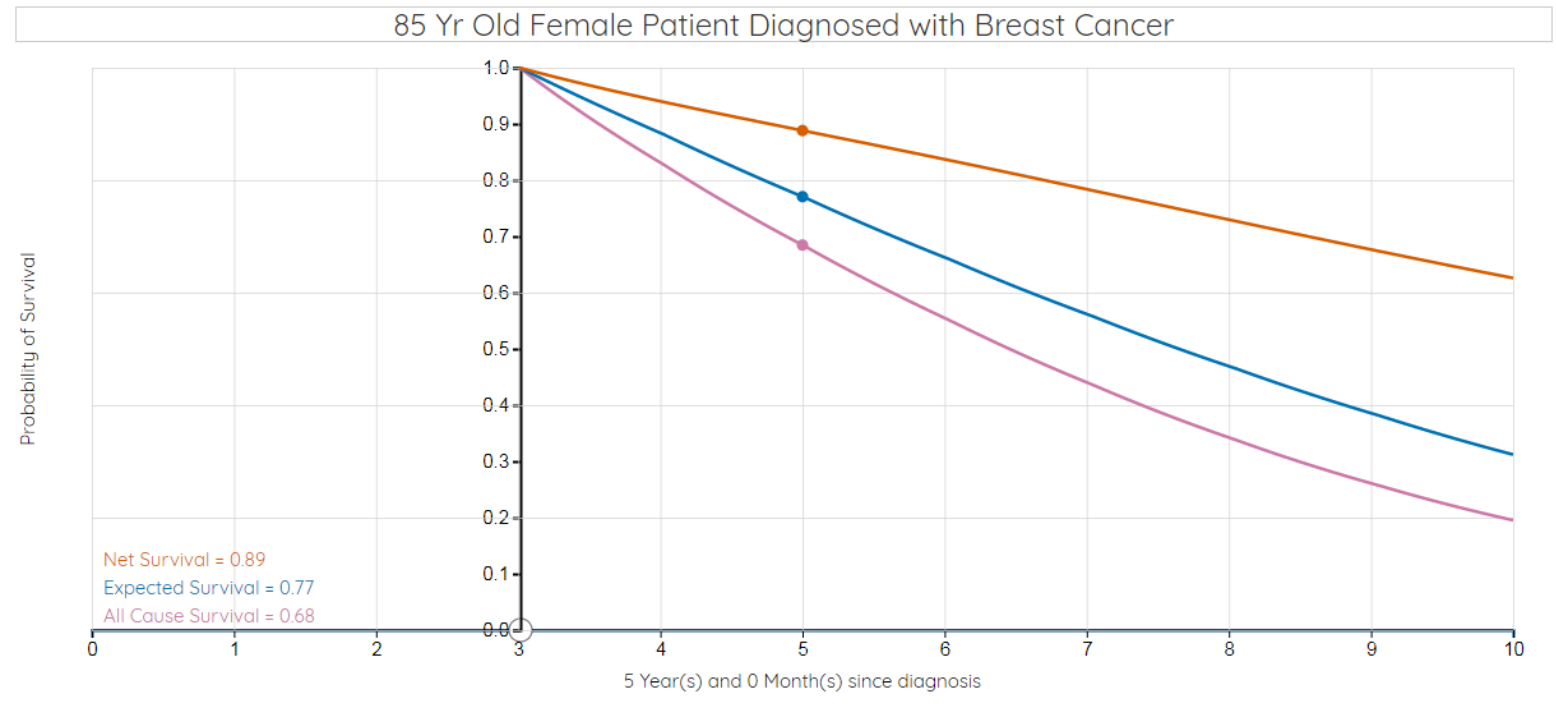

Conditional Survival: Probability of survival if patients are still alive 3 year(s) and 0 month(s) after diagnosis

Figure 1: Screenshot of a line chart giving conditional survival probabilities. Plot represents probabilities of survival for an 85 year old female breast cancer patient if they were still alive 3 years after diagnosis after dragging the $\boldsymbol{y}$-axis.

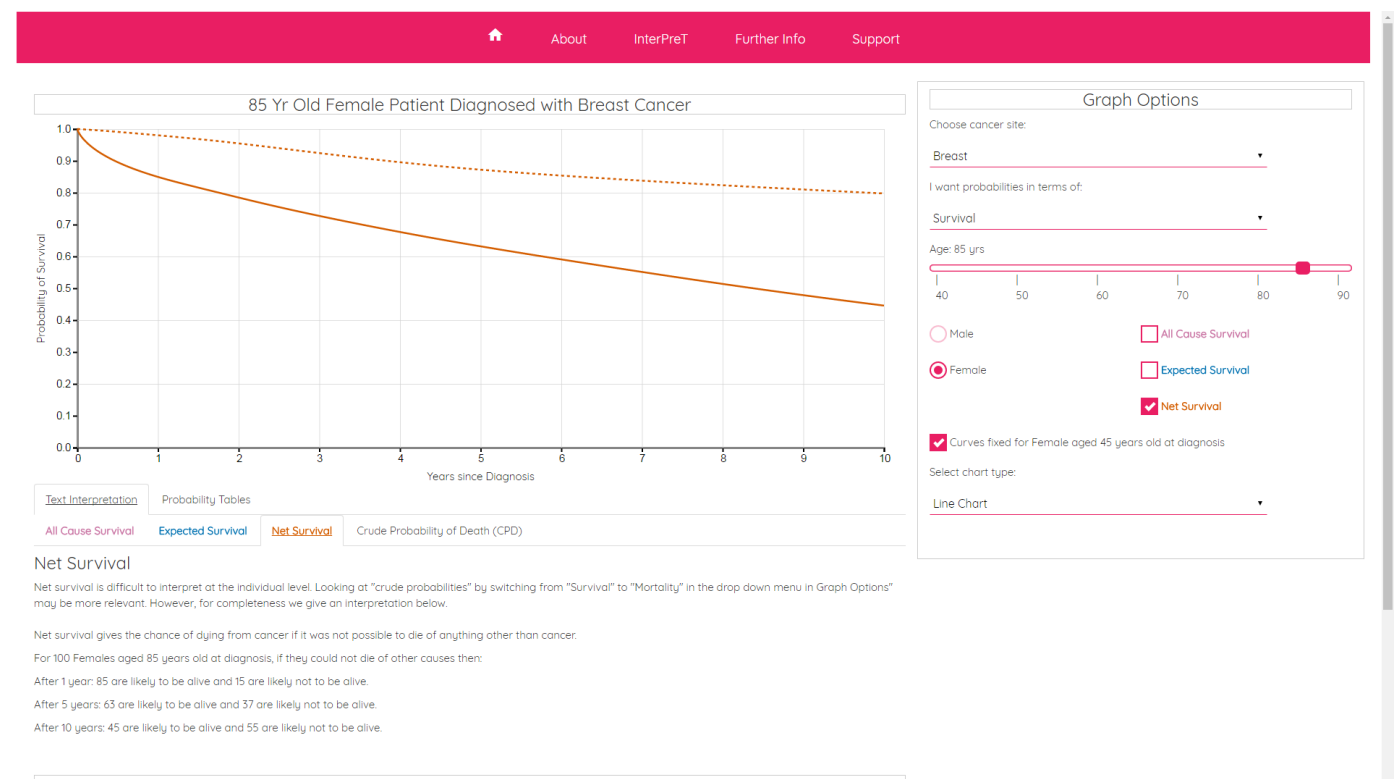

Figure 2: Screenshot of the "InterPreT Cancer Survival" tool page. Illustration of a fixed net survival curve for a 45 year old female breast cancer patient (dashed line) compared to an 85 year old female breast cancer patient (solid line). 
(a) 45 year old female patient

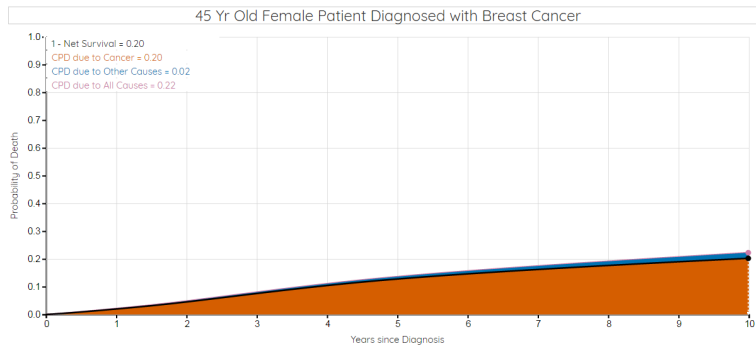

(c) 70 year old female patient

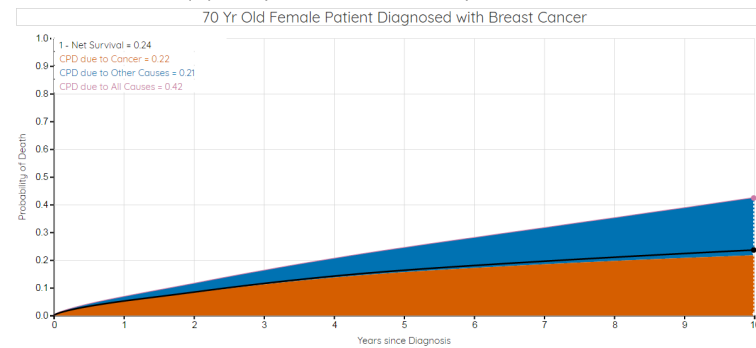

(b) 60 year old female patient

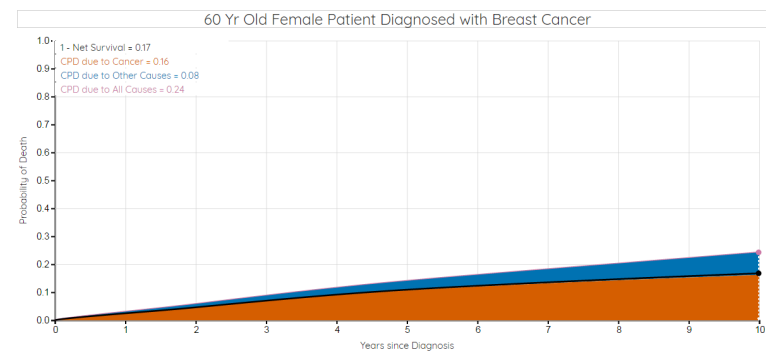

(d) 85 year old female patient

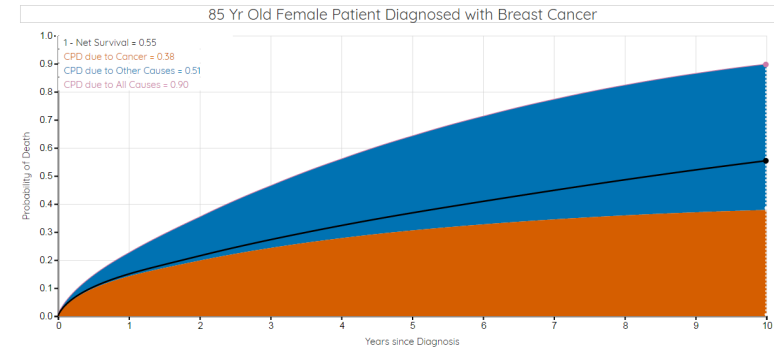

Figure 3: Screenshots of crude probability of death stacked plots for female breast cancer patients at different ages. Orange area refers to the crude probability of death due to cancer and the blue area refers to the crude probability of death due to other causes. The black line compares the net probability of death (1 minus net survival).

(a) 45 year old female patients

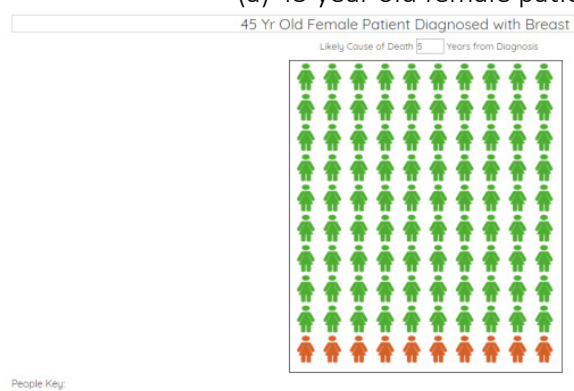

(c) 70 year old female patients

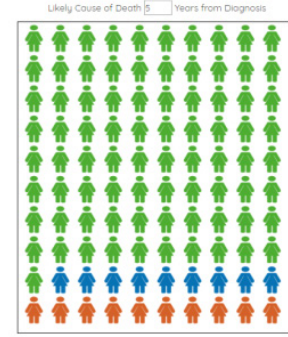

(b) 60 year old female patients

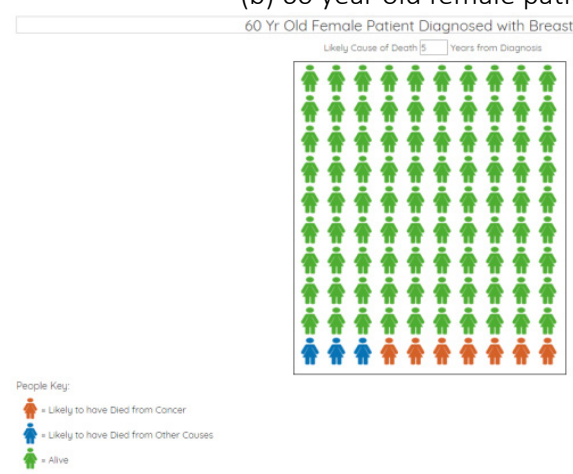

(d) 85 year old female patients

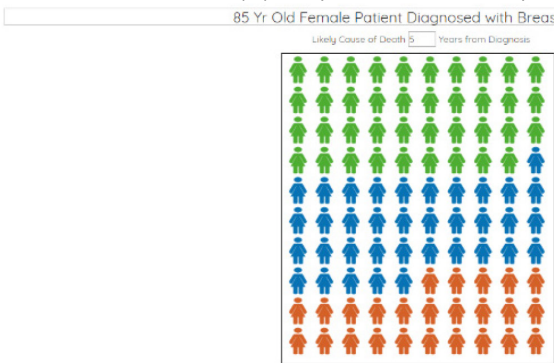

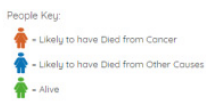

People Key:

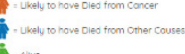

Figure 4: Screenshots of people charts illustrating the crude probability of death. Comparison of crude probability of death due to cancer (orange) and due to other causes (blue) for female breast cancer patients at various ages 5 years after diagnosis. Green people represent patients that are still alive 5 years after diagnosis. 